\title{
BioData Mining I Short Report (Biomedcentral.Com) Combinatorial Analyses Identify A Core Response to the CDX2 Homeoprotein During Development and In Pathologies
}

\author{
Victor GOURAIN \\ Karlsruhe Institute of Technology: Karlsruher Institut fur Technologie \\ Isabelle DULUC \\ INSERM UMR-S1113 / IRFAC \\ Claire DOMON-DELL \\ INSERM UMR-S1113 / IRFAC \\ Jean-Noel FREUND ( $\square$ jean-noel.freund@inserm.fr) \\ INSERM UMR-S1113 / IRFAC https://orcid.org/0000-0002-0971-3774
}

Short report

Keywords: Homeobox gene, embryo, cancer, differential expression, chromosomal targets

Posted Date: January 13th, 2021

DOl: https://doi.org/10.21203/rs.3.rs-143359/v1

License: (c) (i) This work is licensed under a Creative Commons Attribution 4.0 International License.

Read Full License 


\section{Abstract}

Whether a gene involved in distinct tissue or cell functions exerts a core of common molecular activities is a relevant topic in the evolutionary, developmental and therapeutic perspective. Here, we addressed this question by focusing on the CDX2 homeobox gene that encodes a transcription factor with important functions during embryonic development and in adult pathologies. By analysing RNAseq data and ChIPseq data, we unveiled the existence of a core of genes whose expression is responsive to the Cdx2 homeoprotein and also a core of chromosomal regions targeted by $\mathrm{Cdx} 2$ at three important steps of mouse development: trophectoderm formation, posterior body elongation and intestinal specification. In addition, based on human cancer data, we highlighted the relevance of the core of responsive genes by displaying a significant number of human orthologues exhibiting an altered expression along with $C D X 2$ in human malignancies.

\section{Introduction}

That evolution makes new out of old suggests the existence of shared properties between the functions of a given gene at its different times or sites of action. The homeobox gene encoding the $\mathrm{Cdx} 2$ transcription factor allows addressing this assumption since it consecutively drives three major developmental processes in mammals. At the blastula stage, $C d \times 2$ is pivotal for trophectoderm formation during the segregation of pluripotent cells into the first two lineages of trophectodermal cells and inner mass embryonic stem (ES) cells [1,2]; then, $C d x 2$ actively participates in axial posterior body growth at gastrulation [3]; finally, it determines gut identity of the mid- / hindgut embryonic endoderm [4]. In adults, Cdx2 expression is physiologically limited to the gut epithelium, involved in the control intestinal homeostasis [5,6] and endowed with tumor suppressor activity [7-9]. However, it becomes ectopically expressed in precancerous metaplastic lesions and adenocarcinoma of foregut-derived organs, and also in leukemia with an oncogenic role (see [10] for a review and Refs therein). On this basis, the present work interrogates whether some elements of the response to $C d x 2$ are shared at these three developmental steps and subsequently whether these elements are altered in human pathologies along with CDX2.

\section{Materials And Methods}

\section{Data sources}

Mouse RNAseq and ChIPseq raw data were retrieved from the GEO database (https://www.ncbi.nlm.nih.gov/geo/): GSE115541 [11], GSE62149 [12], GSE90752 [13] and GSE84899 [14]. Human RNAseq data from colon adenocarcinoma (COAD), stomach adenocarcinoma (STAD) and acute myeloid leukemia (AML) were obtained from the The Cancer Genome Atlas (https://www.cancer.gov/tcga) (Additional file 2.1). Data for non-dysplastic and low-grade dysplastic esophageal Barrett metaplasia (ESOBM-nd, ESOBM-Igd) and esophageal adenocarcinoma (ESOAD) were from [15] (http://www.ebi.ac.uk/arrayexpress/; E-MTAB-4054). 


\section{Mouse RNAseq data processing}

For mouse RNAseq processing, raw RNAseq reads were quality checked with the FASTX toolkit (http://hannonlab.cshl.edu/fastx_toolkit/index.html) and mapped with STAR [16] against the mouse reference genome GRCm38. Levels of transcripts were computed with HTSeq [17] in union mode, normalized by a regression model with DESeq2 [18] and the consistency of biological replicates was tested by hierarchical clustering [18]. Aberrant levels of transcripts were identified with the Cook distance. Levels of transcripts were normalized by calculation of reads per kilobase per million if no replicate was available. The $p$-values computed with the Wald-test were adjusted with the Bonferroni multiple testing method. Differentially-expressed genes (DEGs) were selected according to the adjusted $p<0.05$ and the $\mid \log _{2}$ (Fold-Change)|>2, and a core of common DEGs was created by comparison among databases. Human orthologues of the mouse DEGs core were identified based on a confidence score of 1 or a minimal sequence homology of $30 \%$, as previously described [19]. Enriched biological functions (GO terms), signalling pathways (KEGG) and protein domains (InterPro) were tested with DAVID [20] and selected with $p<0.05$.

\section{Mouse ChIPseq data processing}

Mouse ChIPseq reads were mapped with BWA [21] against the reference genome GRCm38. Peaks were processed with MACS2 [22] and the signal was normalized by computing fragment pileup per million reads. They were then selected according to $p<0.05$ and a core of peaks was created by comparison among databases for peaks overlapping with at least 10-bp. Intergenic ChIPseq peaks were further compared to Super-Enhancers from the database dbSUPER [23].

\section{DNA binding motifs analysis}

All known DNA binding motifs of vertebrate transcription factors present in the core of ChIPseq peaks and in the gene promoters of the core of DEGs (defined as the 2-kb segment upstream of the canonical transcription start site(s) of each gene) were retrieved from the database JASPAR [24] and classified with TFclass [25]. Enrichments for transcription factor binding motifs was tested with HOMER [26] in the direct vicinity (+/- $50 \mathrm{bp}$ ) of mapped CDX-type binding motifs identified within the promoters of the DEGs and in the overlapping ChIPseq peaks. For this purpose, background sets of DNA sequences were created, composed of the same number of tested regions, i.e. promoters and overlapping ChIPseq peaks. The DNA sequences were of the same size as the tested regions and randomly extracted from the mouse reference genome GRCm38.

\section{Human pathological samples analysis}

For human pathological samples of COAD, STAD and AML, raw levels of transcripts were computed with HTSeq [17] and normalized by computing reads per kilobase per million in order to identify groups of high and low levels of $C D X 2$ transcripts. These groups were defined as upper and lower quartiles or deciles with a purpose of comparable size. For pair-wise comparisons of groups, raw levels of transcripts were 
processed with DESeq2 [18]. Genes with significant variations in transcript levels were selected with adjusted $p<0.05$ (Bonferroni multiple testing method). For ESOBM-nd, ESOBM-Igd and ESOAD, $\| \log _{2}$ (FoldChange)| and $p$-value were retrieved from the literature [15]. Statistical tests for enrichment were performed with the one-tailed exact Fisher test.

\section{Results And Discussion}

\section{A Core of Common Genes Responsive to Cdx2 during Mouse Development}

To address if common genes are regulated by the $\mathrm{Cdx} 2$ transcription factor in its consecutive function during trophectoderm formation, posterior growth and intestinal fate determination in the mouse embryo, RNAseq data were compared between $C d x 2$-overexpressing $v s$ wild type ES cells [12,13], E8 $C d x$-null vs wild type embryos [14] and intestinal epithelial cells of E16 $C d x 2^{-/} v s$ wild type embryos [11]. Using a $\mid \log _{2}$ (Fold-Change) $\mid>2$ and a $p$-value $<0.05$, a core of 221 common differentially-expressed genes (CDEGs) was identified between these 3 conditions, corresponding to 162 orthologue genes in human (Fig. 1a, Additional file 1.1). Interestingly, the direction of the expression changes of the CDEGs, either up or down, was not always similar at the 3 developmental steps, suggesting a context-dependent response to Cdx2 (Fig. 1b, Additional file 1.2). Term enrichment analysis performed on these 162 human orthologues revealed a significant association with "extracellular exosome", "extracellular matrix", "multicellular organism development", "sequence-specific DNA binding", "gene regulation", "metabolic process" and "Wnt signaling" (Fig. 1c, Additional file 1.3). Twenty-eight genes among the 162 orthologues encode nuclear proteins involved in chromatin conformation, DNA transcription and repair, of which 11 are homeobox genes (underlined): Arid3a, Bmyc, $\underline{C d x 1} \underline{C d \times 2}$, Commd3, Ets2, Gata4, Hmgn3, Hoxb1 Hoxb5, Hoxc5 $\underline{\text { Hoxc6 }}, \underline{H o x c 8}$ Id2, Id3, Nkx1.2, Pbx1 Prickle1, Prr13, Pitx1, Rcor2, Smarca1, Sox2, Sp5, Tbx4, Tfeb, TIx2, Znf503.

\section{A Core of Common Chromatin Regions Bound to Cdx2 during Mouse Development}

Next, we compared the location of the $\mathrm{Cdx} 2$ protein on chromatin by ChIPseq at the three developmental steps $[11,13,14]$. This analysis resulted in a core of 1047 common chromosomal regions sharing overlapping peaks in the three conditions (Fig. 1d). Among these peaks, 265 were associated with protein coding genes, 466 with gene promoters (defined as the 2-kb segment upstream of the transcription start site), 52 with non-protein coding genes and their 2-kb promoter, and 264 with intergenic regions (Additional file 1.4). Eight genes of the core of DEGs (Arid3a, Epha4, Hoxc6, Man1c1, Mgat1, Mid1ip1, Sgsm1, Tfeb) exhibited conserved Cdx2 ChIP peak(s) in their 2-kb promoter or coding sequence. This relative low number of CDEGs linked to adjacent Cdx2 ChIPseq peak(s) suggests that important regulatory elements targeted by $\mathrm{Cdx} 2$ might be located far away in intergenic regions. In line with this, 75/264 (28.41\%) of the intergenic Cdx2 ChIPseq peaks fell into Super-Enhancer domains (Additional file 1.5). Alternatively, $C d x 2$ may have both inductive and permissive transcriptional effects, thus uncoupling direct transcription activation from DNA binding, as reported in the gut epithelium [27-29]. Finally, it has 
also been shown that $\mathrm{Cdx} 2$ controls the expression of several downstream targets by interacting with other DNA-binding proteins, without the presence of a typical Cdx-type binding site [30,31].

Sequence analysis showed that 835 of the 1047 ChIPseq regions $(77.75 \%)$ harbored at least one analogous motif to the functionally-described consensus Cdx-binding site (Fig. 1e) [32], giving a total of $1801 \mathrm{Cdx}$-type sites ( $p$-value $=10^{-152}$ ) (Additional file 1.6). Similarly, 214 promoters of the $221 \mathrm{CDGEs}$ (96.83\%) identified above exhibited at least one consensus Cdx-type binding site for a total of 1314 sites (Additional file 1.6). Interestingly, the +/-50-bp segments around the Cdx-type sites present in the ChIPseq regions and in the promoters of the CDEGs were enriched in DNA-binding motifs for respectively 149 transcription factors grouped into 25 families and 74 transcription factors grouped into 11 families ( $p$ value<0.05) (Fig. 1f, Additional files 1.7 and 1.8). The proximity of enriched binding sites for these transcription factors and for $\mathrm{Cdx} 2$ suggests possible direct or indirect interactions.

\section{Fate of the Core of Common CDEGs in Human Pathologies}

Having established the core of 221 CDEGs during mouse embryonic development, we examined human diseases exhibiting alterations in the CDX2 transcript levels for the proportion of the 162 human orthologues deregulated in these pathologies (Fig. 1g, Additional files 2.2). Firstly, given that the CDX2 expression is physiologically limited to the intestine at the adult stage and that it is decreased in a subset of colon adenocarcinoma, we compared the transcriptomes in the deciles of tumors exhibiting the lowest vs highest $C D X 2$ levels ( $n=44$ each) within the TCGA collection of 436 colon adenocarcinoma (COAD). This revealed a significant number of 46 deregulated genes $(p=0.044)$ (Fig. 1h, Additional file 2.3). Conversely, we considered pathological situations exhibiting an abnormal ectopic expression of $C D X 2$ outside the gut, namely in the upper digestive tract, i.e. the esophagus [15] and stomach (TCGA), and in acute myeloid leukemia (TCGA). Retrieving the list of differentially expressed genes between healthy CDX2-free esophageal mucosa and CDX2-expressing non-dysplastic Barrett metaplasia (ESOBM-nd, $\mathrm{n}=14$ ), low-grade dysplastic Barrett metaplasia (ESOBM-lgd, $n=8)$ and adenocarcinoma (ESOAD, $n=12$ ) revealed respectively $123\left(p=0.16 \times 10^{-73}\right), 118\left(p=0.21 \times 10^{-64}\right)$ and $116\left(p=0.96 \times 10^{-44}\right)$ orthologues of the CDEGs core (Fig. 1g,h, Additional files 2.4-6). In the stomach, the list of differentially expressed genes in the quartiles of adenocarcinoma presenting the highest vs lowest levels of $C D X 2$ ( $\mathrm{n}=35$ each) within the series of 272 STOAD samples comprised 44 CDEGs of the core $(p=0.0028)$ (Fig. 1g,h, Additional file 2.7). Similarly, 35 core DEGs $\left(p=0.14 \times 10^{-4}\right)$ were recovered among the genes differentially expressed between the quartiles with the highest vs lowest levels of $C D X 2$ ( $n=38$ each) in the series of $151 \mathrm{AML}$ (Fig $1 \mathrm{~g}, \mathrm{~h}$, Additional file 2.8). Altogether, these data indicate that a significant proportion of genes of the core defined on the basis of mouse developmental models are differentially expressed in human diseases along with CDX2 changes. This proportion was higher when comparing lesions with normal tissues (here, the various types of esophageal lesions) than when comparing tumor samples among them in each pathology (here, colon cancers, stomach cancers and leukemia) because of the variable nature of the specimen in the latter cases.

\section{Conclusion}


The combinatorial analyses of data from mouse embryonic developmental have identified cores of common differentially-expressed genes and chromatin regions responsive to the $\mathrm{Cdx} 2$ homeoprotein at three steps where this transcription factor plays pivotal roles: trophectoderm differentiation, posterior growth of the embryonic body and intestinal determination. Similar functions are anticipated for the CDX2 gene in human embryonic development, because of its specific expression in the trophectoderm lineage at the blastula stage [35], the association of gene mutations with sirenomelia [33], and its abnormal expression linked to gut-type differentiation in congenital endoderm-derived lesions [34]. Beyond embryogenesis, the current results highlight that a significant number of genes of the DEGs core identified in mouse embryos are deregulated in human diseases along with $C D X 2$, which strengthens the relevance of the core in pathologies involving this important developmental gene. Nevertheless, the direction of the changes of several genes of the CDEGs core is not the same at all three developmental stages and also in pathological situations, which emphasizes the context-dependent activity of the CDX2 transcription regulator (Fig. 1b). It can be seen in the light of its anti-repressing effect preventing the incursion of inactive chromatin marks into chromatin domains to keep them accessible for other transcription partners $[28,29]$. Therefore, the outcome of CDX2 might depend not only on the chromatin domains that are kept open, but also on the specific repertoire of nuclear partners present in the cells and able to access the open chromatin regions. Altogether, this study supports the notion that a transcription regulator, while playing distinct functions at different steps during embryonic development, can exert a common subset of molecular activities, and that some of these activities can be subsequently deregulated in adult pathologies along with this factor. This combinatorial approach opens ways to investigate novel functional interactions between developmental genes and to exploit them in a therapeutic perspective.

\section{Abbreviations}

AML: acute myeloid leukemia

CDEGs: common differentially expressed genes

Cdx2: Caudal-related homeobox-2

ChIPseq: chromatin immunoprecipitation sequencing

COAD: colon adenocarcinoma

ESOBM-Igd: low grade dysplastic esophageal Barrett metaplasia

ESOBM-nd: non-dysplastic esophageal Barrett metaplasia

RNAseq: whole transcriptome sequencing

STAD: stomach adenocarcinoma 


\section{Declarations}

\section{Ethics approval and consent to participate}

Not applicable. This work used public data.

\section{Consent for publication}

Not applicable. This work used public data.

\section{Availability of data and materials}

The datasets used for this work are available in public databases:

GEO database (https://www.ncbi.nlm.nih.gov/geo/)

The Cancer Genome Atlas (https://www.cancer.gov/tcga)

The EBI ArrayExpress (http://www.ebi.ac.uk/arrayexpress/).

\section{Competing interests}

The authors declare that they have no competing interests.

\section{Funding}

This work was supported by the IRFAC laboratory (Inserm UMR-S1113) and the Fondation ARC (PJA 20181208021).

\section{Authors' contributions}

J.N.F., V.G., I.D. and C.D-D. conceived the research, analyzed the data and wrote the manuscript; V.G. performed the bioinformatics analyses.

\section{Acknowledgements}

We thank the Inserm for the support of the IRFAC laboratory and the Prof Uwe Strähle, Karlsruhe Institute of Technology, Germany, for providing computing resources.

\section{References}

1. Niwa H, Toyooka Y, Shimosato D, Strumpf D, Takahashi K, Yagi R, et al. Interaction between Oct3/4 and Cdx2 determines trophectoderm differentiation. Cell. 2005;123:917-29.

2. Strumpf D, Mao CA, Yamanaka Y, Ralston A, Chawengsaksophak K, Beck F, et al. Cdx2 is required for correct cell fate specification and differentiation of trophectoderm in the mouse blastocyst.

Development. 2005;132:2093-112. 
3. van Rooijen C, Simmini S, Bialecka M, Neijts R, van de Ven C, Beck F, et al. Evolutionarily conserved requirement of Cdx for post-occipital tissue emergence. Development. 2012;139:2576-83.

4. Gao N, White P, Kaestner KH. Establishment of intestinal identity and epithelial-mesenchymal signaling by Cdx2. Dev Cell. 2009;16:588-99.

5. Stringer EJ, Duluc I, Saandi T, Davidson I, Bialecka M, Sato T, et al. Cdx2 determines the fate of postnatal intestinal endoderm. Development. 2012;139:465-74.

6. Verzi MP, Shin H, Ho L-L, Liu XS, Shivdasani RA. Essential and redundant functions of caudal family proteins in activating adult intestinal genes. Mol Cell Biol. 2011;31:2026-39.

7. Bonhomme C, Duluc I, Martin E, Chawengsaksophak K, Chenard MP, Kedinger M, et al. The Cdx2 homeobox gene has a tumour suppressor function in the distal colon in addition to a homeotic role during gut development. Gut. 2003;52:1465-71.

8. Sakamoto N, Feng Y, Stolfi C, Kurosu Y, Green M, Lin J, et al. BRAFV600E cooperates with CDX2 inactivation to promote serrated colorectal tumorigenesis. Elife. 2017;6:e20331.

9. Balbinot C, Armant O, Elarouci N, Marisa L, Martin E, De Clara E, et al. The Cdx2 homeobox gene suppresses intestinal tumorigenesis through non-cell-autonomous mechanisms. J Exp Med. 2018;215:911-26.

10. Chawengsaksophak K. Cdx2 Animal Models Reveal Developmental Origins of Cancers. Genes (Basel). 2019;10:928.

11. Banerjee KK, Saxena M, Kumar N, Chen L, Cavazza A, Toke NH, et al. Enhancer, transcriptional, and cell fate plasticity precedes intestinal determination during endoderm development. Genes Dev. 2018;32:1430-42.

12. Cambuli F, Murray A, Dean W, Dudzinska D, Krueger F, Andrews S, et al. Epigenetic memory of the first cell fate decision prevents complete ES cell reprogramming into trophoblast. Nat Commun. 2014;5:5538.

13. Rhee C, Lee B-K, Beck S, LeBlanc L, Tucker HO, Kim J. Mechanisms of transcription factor-mediated direct reprogramming of mouse embryonic stem cells to trophoblast stem-like cells. Nucleic Acids Res. 2017;45:10103-14.

14. Amin S, Neijts R, Simmini S, van Rooijen C, Tan SC, Kester L, et al. Cdx and T Brachyury Co-activate Growth Signaling in the Embryonic Axial Progenitor Niche. Cell Rep. 2016;17:3165-77.

15. Maag JLV, Fisher OM, Levert-Mignon A, Kaczorowski DC, Thomas ML, Hussey DJ, et al. Novel Aberrations Uncovered in Barrett's Esophagus and Esophageal Adenocarcinoma Using Whole Transcriptome Sequencing. Mol Cancer Res. 2017;15:1558-69.

16. Dobin A, Davis CA, Schlesinger F, Drenkow J, Zaleski C, Jha S, et al. STAR: ultrafast universal RNAseq aligner. Bioinformatics. 2013;29:15-21.

17. Anders S, Pyl PT, Huber W. HTSeq--a Python framework to work with high-throughput sequencing data. Bioinformatics. 2015;31:166-9. 
18. Love MI, Huber W, Anders S. Moderated estimation of fold change and dispersion for RNA-seq data with DESeq2. Genome Biol. 2014;15:550.

19. Mayrhofer M, Gourain V, Reischl M, Affaticati P, Jenett A, Joly J, et al. A novel brain tumour model in zebrafish reveals the role of YAP activation in MAPK- and PI3K-induced malignant growth. Disease models \& mechanisms. 2017;10:15-28.

20. Huang DW, Sherman BT, Lempicki RA. Systematic and integrative analysis of large gene lists using DAVID bioinformatics resources. Nat Protoc. 2009;4:44-57.

21. Li H, Durbin R. Fast and accurate short read alignment with Burrows-Wheeler transform. Bioinformatics. 2009;25:1754-60.

22. Zhang Y, Liu T, Meyer CA, Eeckhoute J, Johnson DS, Bernstein BE, et al. Model-based analysis of ChIP-Seq (MACS). Genome Biol. 2008;9:R137.

23. Khan A, Zhang X. dbSUPER: a database of super-enhancers in mouse and human genome. Nucleic Acids Res. 2016;44:D164-171.

24. Khan A, Fornes O, Stigliani A, Gheorghe M, Castro-Mondragon JA, van der Lee R, et al. JASPAR 2018: update of the open-access database of transcription factor binding profiles and its web framework. Nucleic Acids Res. 2018;46:D260-6.

25. Wingender E, Schoeps T, Haubrock M, Krull M, Dönitz J. TFClass: expanding the classification of human transcription factors to their mammalian orthologs. Nucl Acids Res. 2018;46:D343-7.

26. Heinz S, Benner C, Spann N, Bertolino E, Lin YC, Laslo P, et al. Simple combinations of lineagedetermining transcription factors prime cis-regulatory elements required for macrophage and $B$ cell identities. Mol Cell. 2010;38:576-89.

27. San Roman AK, Tovaglieri A, Breault DT, Shivdasani RA. Distinct Processes and Transcriptional Targets Underlie CDX2 Requirements in Intestinal Stem Cells and Differentiated Villus Cells. Stem Cell Reports. 2015;5:673-81.

28. Saxena M, Roman AKS, O'Neill NK, Sulahian R, Jadhav U, Shivdasani RA. Transcription factordependent "anti-repressive" mammalian enhancers exclude H3K27me3 from extended genomic domains. Genes Dev. 2017;31:2391-404.

29. Verzi MP, Shin H, San Roman AK, Liu XS, Shivdasani RA. Intestinal master transcription factor CDX2 controls chromatin access for partner transcription factor binding. Mol Cell Biol. 2013;33:281-92.

30. Kim S-P, Park J-W, Lee S-H, Lim JH, Jang B-C, Lee S-H, et al. Homeodomain protein CDX2 regulates COX-2 expression in colorectal cancer. Biochem Biophys Res Commun. 2004;315:93-9.

31. Mutoh $\mathrm{H}$, Hayakawa $\mathrm{H}$, Sakamoto $\mathrm{H}$, Sugano K. Homeobox protein CDX2 reduces Cox-2 transcription by inactivating the DNA-binding capacity of nuclear factor-kappaB. J Gastroenterol. 2007;42:71929.

32. Margalit $Y$, Yarus $S$, Shapira E, Gruenbaum Y, Fainsod A. Isolation and characterization of target sequences of the chicken CdxA homeobox gene. Nucl Acids Res. 1993;21:4915-22. 
33. Lecoquierre F, Brehin A-C, Coutant S, Coursimault J, Bazin A, Finck W, et al. Exome sequencing identifies the first genetic determinants of sirenomelia in human. Hum Mutat. 2020;41:926-33.

34. Martin E, Vanier M, Tavian M, Guerin E, Domon-Dell C, Duluc I, et al. CDX2 in congenital gut gastrictype heteroplasia and intestinal-type Meckel diverticula. Pediatrics. 2010;126:e723-727.

35. Yan L, Yang M, Guo H, Yang L, Wu J, Li R, et al. Single-cell RNA-Seq profiling of human preimplantation embryos and embryonic stem cells. Nat Struct Mol Biol. 2013;20:1131-9.

\section{Figures}


a

b

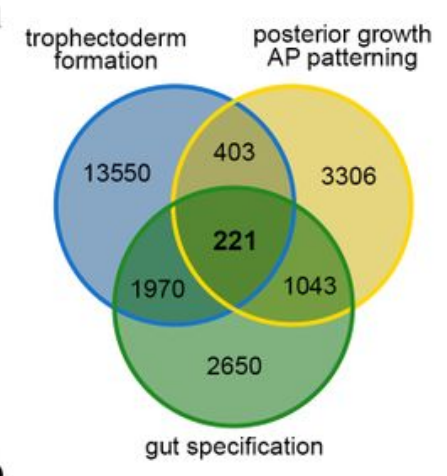

C

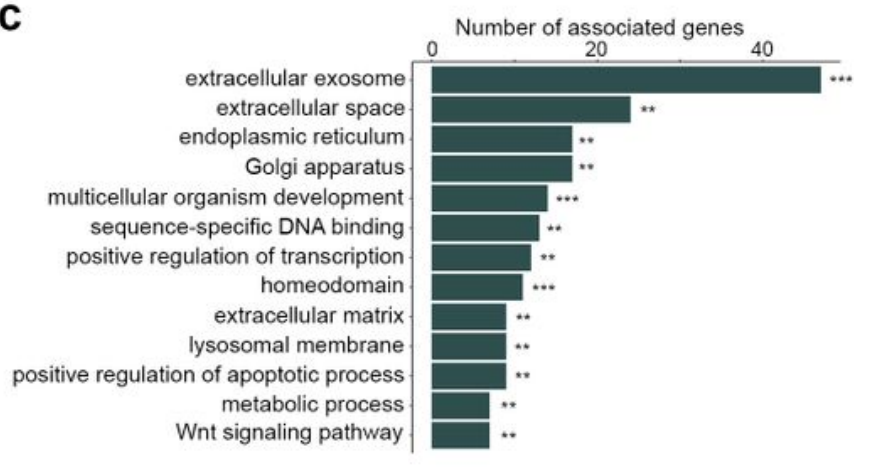

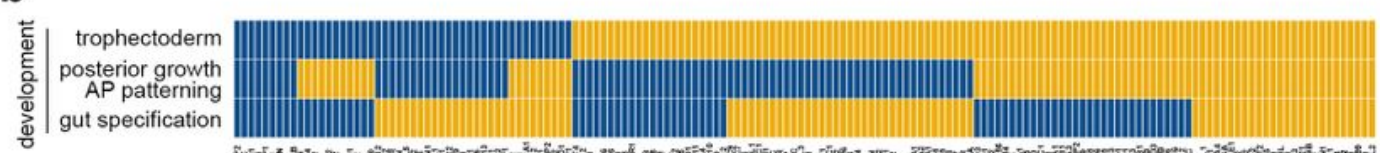

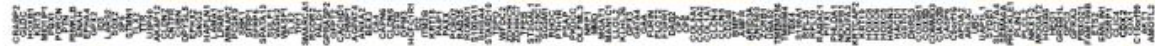

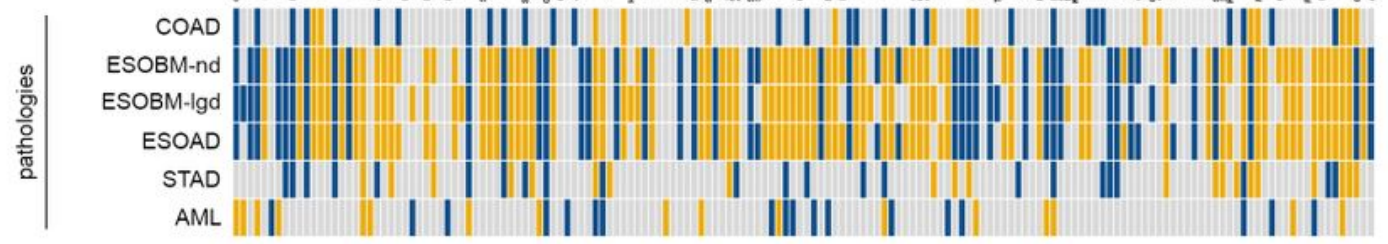

d

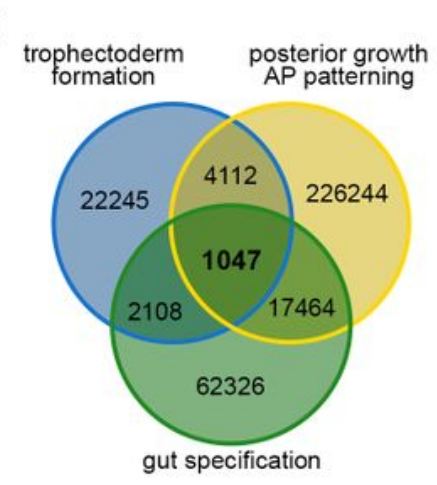

$f_{\frac{g}{5}}$

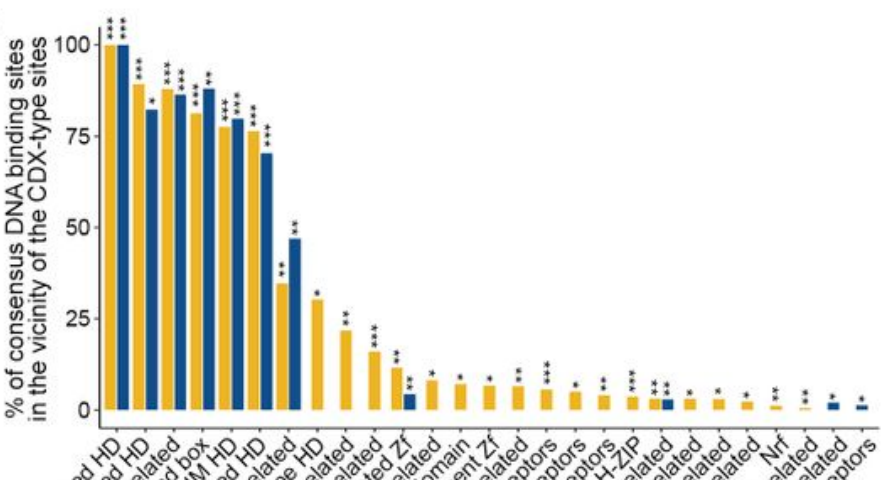

e

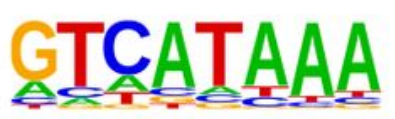

g
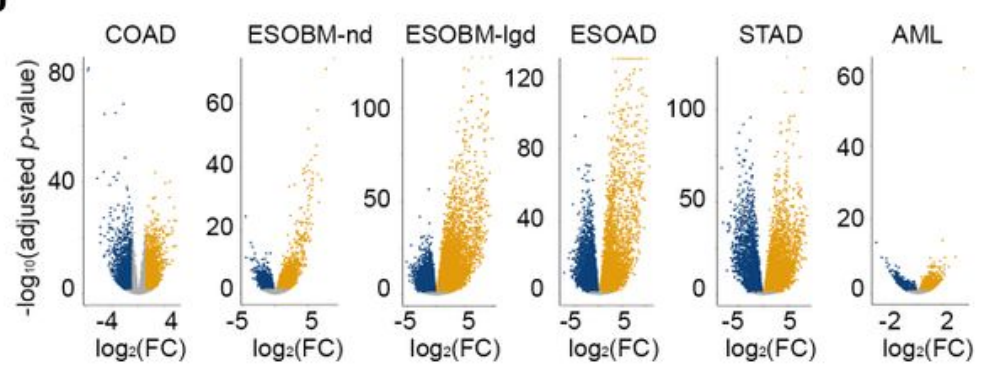

h

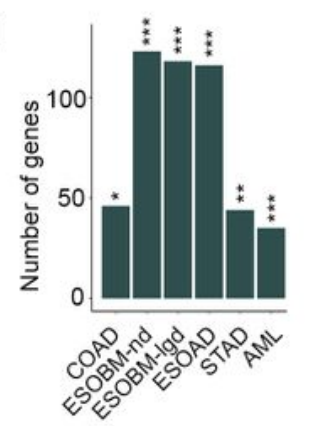

\section{Figure 1}

The core of CDX2 targets. (a) The core of $221 \mathrm{CDEGs}$ between Cdx2-overexpressing vs wildtype ES cells (blue), E8 Cdx-null vs wildtype embryos (yellow) and intestinal epithelial cells of E16 Cdx2-/-vs wildtype embryos (green) (see also Additional file 1.1). (b) Correlation map between changes of the 162 human orthologues to the mouse CDEGs core during development (upper panel) and pathologies (lower panel); up: yellow; down: blue (Additional files 1.2 and 2.3-8). (c) Term enrichment analysis of the human 
orthologues. ${ }^{* \star} p<0.01,{ }^{* \star \star} p<0.001$ (Additional file 1.3). (d) The 1047 common Cdx2 ChIP regions during trophectoderm formation (blue), embryonic posterior elongation (yellow) and gut specification (green) (Additional file 1.4). (e) Consensus sequence of the Cdx-type motifs present in the Cdx2 ChIP regions and in the promoters of the CDEGs (Additional file 1.6). (f) Enrichment in consensus binding motifs for the indicated transcription factors families in the vicinity (+/-50 bp) of the Cdx-type sites present in the 1047 ChIP regions (yellow) and in the promoters of the 221 CDEGs (blue). ${ }^{*} p<0.05,{ }^{\star *} p<0.01,{ }^{\star * \star} p<0.00001$. HD: homeodomain; Zf: zinc finger; h: hormone (Additional files 1.7-8). (g) Differentially expressed genes in colon cancers (COAD), in esophageal non-dysplastic Barrett metaplasia (ESOBM-nd), low-grade dysplastic Barrett metaplasia (ESOBM-Igd) and adenocarcinoma (ESOAD), in gastric adenocarcinoma (STAD) and in acute myeloid leukemia (AML); each spot corresponds to one gene; up: yellow, down: blue (Additional file 2.2). (h) Number of human orthologues deregulated along with CDX2 in the indicated diseases. ${ }^{\star} p<0.05,{ }^{* \star} p<0.001,{ }^{* \star *} p<0.0001$ (Additional files 2.3-8).

\section{Supplementary Files}

This is a list of supplementary files associated with this preprint. Click to download.

- Additionalfile1.xls

- Additionalfile2.xls 